\title{
Conjugative Transposition of Tn916 and Detection of Tn916-Like Transposon in Erysipelothrix rhusiopathiae
}

\author{
Manao OZAWA ${ }^{1)}$, Kinya YAMAMOTO ${ }^{1)}$, Akemi KOJIMA ${ }^{1)}$, Masami TAKAGI ${ }^{1)}$ and Toshio TAKAHASHI ${ }^{1)}$ \\ ${ }^{1)}$ National Veterinary Assay Laboratory, Ministry of Agriculture, Forestry and Fisheries, 1-15-1 Tokura, Kokubunji, Tokyo 185-8511, Japan
}

(Received 1 October 2008/Accepted 12 July 2009)

\begin{abstract}
In order to investigate the origin of tetracycline resistance in Erysipelothrix rhusiopathiae, conjugative transpositions of Tn916 were tested. The frequency of transfer between strains of E. rhusiopathiae was about 10-fold higher than that between Enterococcus faecalis and E. rhusiopathiae. In addition, detection of a Tn916-like transposon was performed by PCR assay and DNA sequencing in E. rhusiopathiae field isolates. Of 49 tetracycline-resistant isolates, 38 (77.6 \%) carried a Tn916-like transposon, while $11(22.4 \%)$ carried $\operatorname{tet}(\mathrm{M})$ only. These results suggested that Tn916-like transposon may be widely present in the E. rhsuiopathiae field isolates resistant to tetracycline.
\end{abstract}

KEY WORDS: antimicrobial resistance, Erysipelothrix rhusiopathiae, tetracycline, $\operatorname{Tn} 916$.

Erysipelothrix rhusiopathiae is the causative agent of swine erysipelas, which causes great economic loss in pig husbandry throughout the world. E. rhusiopathiae is also an important pathogen in public health as it causes erysipeloid in humans [19]. The tetracycline group of antibiotics is frequently used for growth promotion or treatment of various infections in pigs. The emergence of strains resistant to oxytetracycline (OTC) was first reported in 1984 [16]. Recently, Yamamoto et al. [20] demonstrated that $53.3 \%$ of E. rthusiopathiae strains isolated from 1988 to1998 were resistant to OTC. They detected the tet(M) gene in E. rhusiopathiae and indicated that the nucleotide sequence of the tet $(\mathrm{M})$ gene had $99 \%$ identity with the tet $(\mathrm{M})$ gene from Tn916 of Enterococcus faecalis [21]. Tn916, initially detected in E. faecalis, is a broad-host-range conjugative transposon that carries the $\operatorname{tet}(\mathrm{M})$ gene $[5,15]$. Tn916 and related elements are found in a wide variety of Gram-positive and a few Gram-negative bacterial species [13]. It has been recognized that $\mathrm{Tn} 916$ is transferable from Enterococus and Listeria to Erytipelothrix by in vitro conjugation $[14,17]$. In this study, we investigated conjugative transposition from E. rhusiopathiae to E. rhuisiopathiae and distribution of Tn916-like transposon in E. rhusiopathiae field isolates, to clarify how tet( $\mathrm{M})$ gene is spread in E. rhusiopathiae strains in the field.

The main characteristics of the bacterial strains used in this study are listed in Table 1. The strain Fujisawa of $E$. rhusiopathiae was used as a recipient [9]. Tn916 was transposed from E. faecalis CG110 [6] to E. faecalis OG1X [7] and this strain was designated OG1X::Tn916 and used as the donor for $\operatorname{Tn} 916$. The spontaneous mutant strains resistant to dihydrostreptomycin (DSM) were obtained from a bacterial suspension in tryptose phosphate broth (TPB;

\footnotetext{
* Correspondence to: Ozawa, M., National Veterinary Assay Laboratory, Ministry of Agriculture, Forestry and Fisheries, 1-15-1, Tokura, Kokubunji, Tokyo 185-8511, Japan.

e-mail: ozawa@nval.go.jp
}

Difco Laboratories, MI, U.S.A.) that contained $0.1 \%$ Tween 80 (pH 7.6) (TPB-T80) and dihydrostreptomycin (100 $\mu \mathrm{g} /$ $\mathrm{ml}$ ). These mutant strains were designated T17-DSMR and C4-DSMR. In detection of Tn916-like transposon, $68 \mathrm{E}$. rhusiopathiae field isolates were used. They were isolated from cases of swine erysipelas between 1994 and 2001 in Japan. Of 68 isolates, 49 (72.1\%) were OTC-resistant [minimum inhibitory concentration (MIC) range, 16-64 $\mu \mathrm{g} / \mathrm{m} l)]$. MICs were determined as described previously [8].

Filter matings were carried out as follows. Overnight cultures of donor and recipient cells in TPB-T80 broth were mixed at a ratio of $1: 10$. One millilitre of the mating mixture was inoculated on a membrane filter placed on TPB-T80 agar. After $20 \mathrm{hr}$ incubation at $37^{\circ} \mathrm{C}$, the cells were suspended in $2 \mathrm{ml}$ TPB-T80 broth and plated on agar that contained appropriate antibiotics to select transconjugants. In filter matings between $E$. faecalis and E. rhusiopathiae, agar containing kanamycin $(250 \mu \mathrm{g} / \mathrm{m} l)$ and OTC $(10 \mu \mathrm{g} / \mathrm{m} l)$ was used. In filter matings between strains of E. rhusiopathiae, agar containing DSM $(100 \mu \mathrm{g} / \mathrm{m} l)$ and OTC $(10 \mu \mathrm{g} / \mathrm{m} l)$ was used. PCR for termini of circular intermediate of Tn916

Table 1. Bacterial strains used in this study

\begin{tabular}{llc}
\hline Strains & Relevant properties & Reference \\
\hline E. faecalis & & \\
CG110 & OTC $^{\mathrm{r}}$ & 6 \\
OG1X & SM $^{\mathrm{r}}$ & 7 \\
OG1X::Tn916 & OTC $^{\mathrm{r}}, \mathrm{SM}^{\mathrm{r}}$ & This study \\
E. rhusiopathiae & & \\
Fujisawa & Wild type (serotype 1a) & 9 \\
Fujisawa::Tn916 & OTC $^{\mathrm{r}}$ & This study \\
T17 & Wild type (serotype 2) $^{\text {C4 }}$ & This study \\
T17-DSMR & Wild type (serotype2) $^{\mathrm{r}}$ (spontaneous mutant) & This study \\
C4-DSMR & DSM $^{\mathrm{r}}$ (spontaneous mutant) & This study \\
\hline
\end{tabular}

$\mathrm{OTC}^{\mathrm{r}}$, oxytetracycline resistant; $\mathrm{SM}^{\mathrm{r}}$, streptomycin resistant; $\mathrm{DSM}^{\mathrm{r}}$, dihydrostreptomycin resistant. 


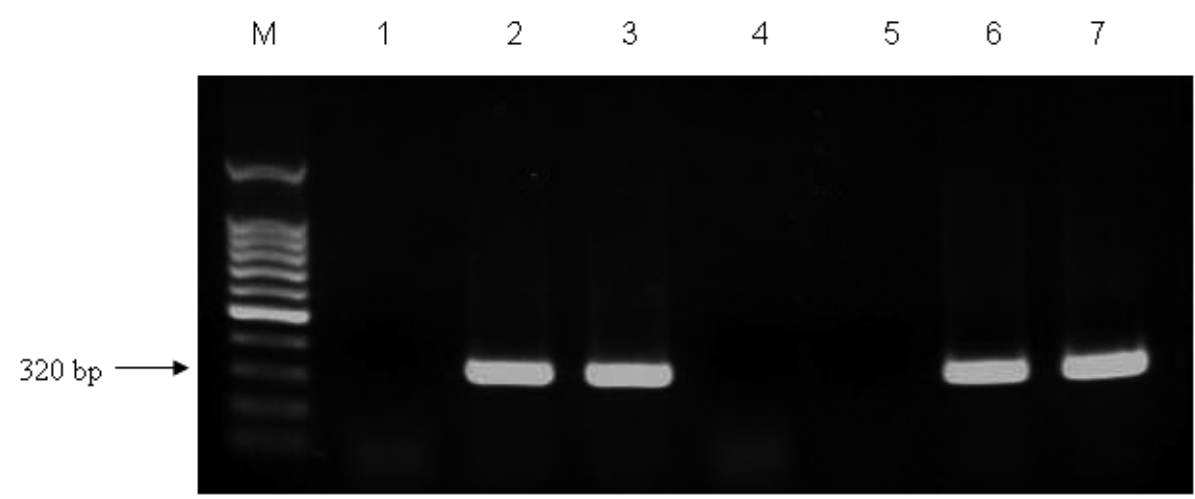

Fig. 1. Electrophoretic analysis of PCR products $(320 \mathrm{bp})$ obtained by amplification of primers designed on Tn916 termini. Lane M, DNA size marker (100 bp ladder), lane 1, Fujisawa, lane 2, OG1X:Tn916, lane 3, Fujisawa:Tn916, lane 4, T17-DSMR, lane 5, C4-DSMR, lane 6, T17DSMR:Tn916, lane 7, C4-DSMR:Tn916.

were performed to confirm conjugative transposition of Tn916. DNA extraction of E. faecalis and E. rhusiopathiae strains was performed as previously described [10]. PCR primers were designed on Tn916 termini [11]. Their positions in the Tn916 nucleotide sequence [4] (GenBank Accession No. U09422) were as follows: Tn916-F1, 5'AACAGGAGCGTCTTGTTGCT-3' (17836-17855); Tn916-R1, 5'-CGTCGTATCAAAGCTCATTCA-3' (11797). The PCR mixture consisted of $100 \mathrm{ng}$ template DNA, 10 pmol each primer, $1 \times$ PCR buffer $\left(\mathrm{Mg}^{2+}\right.$ free $), 2.5 \mathrm{mM}$ $\mathrm{MgCl}_{2}, 0.2 \mathrm{mM}$ dNTP mixture, and $1.25 \mathrm{U}$ Ex Taq DNA polymerase (TaKaRa Ex Taq; Takara Bio Inc., Shiga, Japan). DNA amplification was carried out as follows: 5 min at $94^{\circ} \mathrm{C}$ followed by 35 cycles of $1 \mathrm{~min}$ at $94^{\circ} \mathrm{C}, 1 \mathrm{~min}$ at $56^{\circ} \mathrm{C}, 1 \mathrm{~min}$ at $72^{\circ} \mathrm{C}$ and a final extension of $10 \mathrm{~min}$ at $72^{\circ} \mathrm{C}$. The PCR products were electrophoresed through a $1 \%$ agarose gel and visualized by ethidium bromide staining. The circular forms of Tn916 could be detected in the transconjugants, whereas they were not present in the parent strains Fujisawa, T17-DSMR and C4-DSMR (Fig. 1). The frequencies of transfer are shown in Table 2. In the filter mating using E. faecalis OG1X::Tn916 as donor with E. rhusiopathiae Fujisawa as recipient, the frequency of transfer was $10^{-6}$. On the other hand, in the filter matings using Fugisawa::Tn916 as donor with T17-DSMR and C4-DSMR as recipients, the frequencies of transfer was $10^{-5}$. The frequency of transfer between strains of E. rhusiopathiae was about 10-fold higher than that between $E$. faecalis and $E$. rhusiopathiae.

Detection of tet(M) gene and Tn916-like transposon in the E. rhusiopathiae field isolates was performed using PCR methods as previously described $[1,18]$. PCR products were purified using the QIAquick PCR purification kit (QIAGEN, Valencia, CA, U.S.A.) for use in sequencing reactions. Dye terminator cycle sequencing was performed with the BigDye Terminator Cycle sequencing kit and ABI PRISM 310 genetic analyzer (Applied Biosystems). The restriction enzyme HincII (Takara Bio Inc.) was used to
Table 2. Conjugative transfer frequency of $\operatorname{Tn} 916$

\begin{tabular}{llc}
\hline Donor & Receipient & $\begin{array}{c}\text { Transposon transfer frequency } \\
\text { (transconjugants/donor) }\end{array}$ \\
\hline OG1X::Tn916 & Fujisawa & $7.8 \times 10^{-6}$ \\
Fujisawa::Tn916 & T17-DSMR & $2.3 \times 10^{-5}$ \\
Fujisawa::Tn916 & C4-DSMR & $7.6 \times 10^{-5}$ \\
\hline
\end{tabular}

evaluate the specificity of the PCR assay for the detection of Tn916-like transposon. Of the 49 OTC-resistant isolates, 38 (77.6\%) gave the expected PCR products of $2835 \mathrm{bp}$ using primers linking tet $(\mathrm{M})$ to Xis-Tn genes of Tn916. Of the seven isolates with low MIC values $(16 \mu \mathrm{g} / \mathrm{m} l)$, six $(85.7 \%)$ did not have a Tn916. All of the OTC-resistant E. rhusiopathiae isolates had tet $(\mathrm{M})$. The parts of the PCR product linking tet $(\mathrm{M})$ to Xis-Tn genes of Tn916 from four OTCresistant field isolates were sequenced and had $99.8 \%$ and $100 \%$ homology with Tn916 of E. faecalis respectively (U09422, positions 13907-14337 and 15882-16401). They also had $99.8 \%$ homology with Tn916 of Streptococcus suis (EF432727, positions 16637-17067 and 18611-19130). To evaluate the specificity of the PCR assay for detection of the Tn916-like transposon, the amplified DNA fragments of OTC-resistant E. rhusiopathiae strains and E. faecalis OG1X::Tn916 were digested with Hinc II. All of the amplified DNA fragments digested with Hinc II generated two fragments (1623 and 1212) (Fig. 2).

The tet $(\mathrm{M})$ gene is common in Enterococcus and Streptococcus [22] and has also been found in Listeria [2, 12]. Yamamoto et al. [21] identified the tet(M) gene in the field isolates of E. rhusiopathiae. The wide distribution of this gene may occur because it is carried by broad-host range conjugative transposons that belong to the $\operatorname{Tn} 916$ family [13]. Our results suggest that the presence of tet(M) in $E$. rhusiopathiae is the outcome of acquisition of a Tn916 from Enterococcus. This proposal is based on the fact that $\operatorname{Tn} 916$ is transferable from Enterococcus and Listeria to E. rhusiopathiae by in vitro conjugation $[14,17]$. In addition, Tn916 


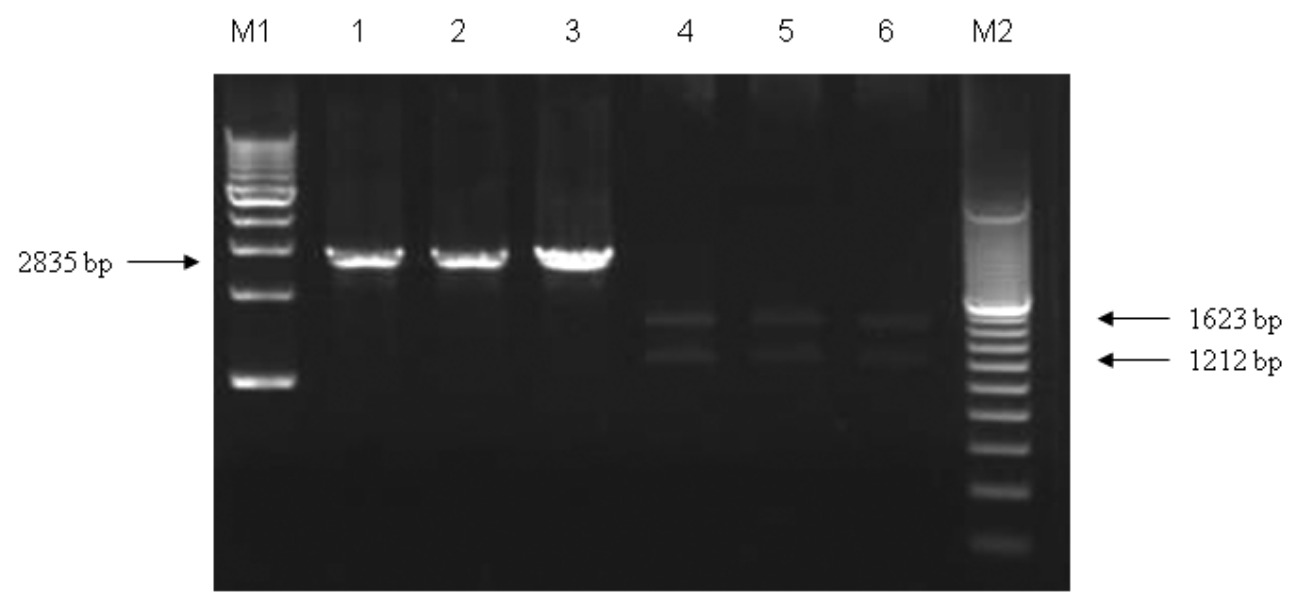

Fig. 2. The specificity of the PCR assay for the detection of the Tn916-like transposon. Lane M1, DNA size marker (1 kb ladder); lane 1, DNA fragment from E. faecalis OG1X::Tn916 (2,835 bp); lane 2, DNA fragment from E. rhusiopathiae T1 (oxytetracycline-resistant field isolate) $(2,835 \mathrm{bp})$; lane 3, DNA fragment from E. rhusiopathiae C16 (oxytetracycline-resistant field isolate)(2,835 bp); lane 4, Hinc II-digested DNA fragment from E. faecalis OG1X::Tn916 (1,623 bp and 1,212 bp); lane 5, Hinc II-digested DNA fragment from $E$. rhusiopathiae T1 (1,623 bp and 1,212 bp); lane 6, Hinc II-digested DNA fragment from $E$. rhusiopathiae C16 (1,623 bp and 1,212 bp); lane M2, DNA size marker (200 bp ladder).

was also transferred between strains of E. rhusiopathiae in this study. The nucleotide sequence of the $\operatorname{tet}(\mathrm{M})$ gene from E. rhusiopathiae had $99 \%$ identity with that from $\operatorname{Tn} 916$ of E. faecalis [21]. Moreover, most OTC-resistant E. rhusiopathiae had a Tn916-like transposon. Streptococcus may also be a donor of $\operatorname{Tn} 916$ because of high similarity of sequence of $\operatorname{Tn} 916$ between E. rhusiopathiae and S. suis in this study. On the other hand, $22.4 \%$ of tetracycline-resistant isolates did not have Tn916 but they had $\operatorname{tet}(\mathrm{M})$ gene. Conjugative plasmids or transposons other than Tn916 may involved in the acquisition of tet $(\mathrm{M})$ gene in these isolates.

Doucet-Populaire et al. [3] have demonstrated the transfer of $\operatorname{Tn} 1545$ from E. faecalis to Listeria monocytogenes in the digestive tract of gnotobiotic mice. Enterococcus and Streptococcus live naturally in the intestinal tract of pigs. The digestive tract is considered to be the most probable meeting point for these two bacterial species and E. rhusiopathiae. Therefore, the interspecific transfer of $\operatorname{Tn} 916$ might occur in the digestive tract.

In conclusion, we demonstrated conjugative transposition of Tn916 between E. rhusiopathiae strains and detected Tn916-like transposon in OTC-resistant E. rhusiopathiae field isolates. Further investigation of transfer of Tn916 in vivo is needed to clarify the reason of wide distribution of tet(M) gene in E. rhusiopathiae strains.

\section{REFERENCES}

1. Agersø, Y., Jensen, L.B., Givskov, M. and Roberts, M.C. 2002. The identification of a tetracycline resistance gene tet(M), on a Tn916-like transposon, in the Bacillus cereus group. FEMS Microbiol. Lett. 214: 251-256.

2. Charpentier, E., Gerbaud, G., Jacquet, C., Rocourt, J. and
Courvalin, P. 1995. Incidence of antibiotic resistance in Listeria species. J. Infect. Dis. 172: 277-281.

3. Doucet-Populaire, F., Trieu-Cuot, P., Dosbaa, I., Andremont, A. and Courvalin, P. 1991. Inducible transfer of conjugative transposon Tn 1545 from Enterococcus faecalis to Listeria monocytogenes in the digestive tracts of gnotobiotic mice. Antimicrob. Agents Chemother. 35: 185-187.

4. Flannagan, S.E., Zitzow, L.A., Su, Y.A. and Clewell, D.B. 1994. Nucleotide sequence of the $18-\mathrm{kb}$ conjugative transposon Tn916 from Enterococcus faecalis. Plasmid 32: 350-354.

5. Franke, A.E. and Clewell, D.B. 1981. Evidence for a chromosome-borne resistance transposon (Tn916) in Streptococcus faecalis that is capable of "conjugal" transfer in the absence of a conjugative plasmid. J. Bacteriol. 145: 494-502.

6. Gawron-Burke, C. and Clewell, D.B. 1982. A transposon in Streptococcus faecalis with fertility properties. Nature 300: 281-284.

7. Ike, Y., Craig, R.A., White, B.A., Yagi, Y. and Clewell, D.B. 1983. Modification of Streptococcus faecalis sex pheromones after acquisition of plasmid DNA. Proc. Natl. Acad. Sci. U.S.A. 80: $5369-5373$.

8. Ishiyama, S., Ueda, S., Kuwabara, N., Kosakai, G., Koya, M., Konno, M. and Fujii, R. 1968. On the standardization of method for determination of minimum inhibitory concentrations. Chemotherapy (Tokyo) 16: 98-99.

9. Kucsera, G. 1972. Comparative study on special serotypes of Erysipelothrix rhusiopathiae strains isolated in Hungary and abroad. Acta Vet. Acad. Sci. Hung. 22: 251-261.

10. Makino, S., Okada, Y., Maruyama, T., Ishikawa, K., Takahashi, T., Nakamura, M., Ezaki, T. and Morita, H. 1994. Direct and rapid detection of Erysipelothrix rhusiopathiae DNA in animals by PCR. J. Clin. Microbiol. 32: 1526-1531.

11. Manganelli, R., Romano, L., Ricci, S., Zazzi, M. and Pozzi, G. 1995. Dosage of Tn916 circular intermediates in Enterococcus faecalis. Plasmid 34: 48-57.

12. Poyart-Salmeron, C., Trieu-Cuot, P., Carlier, C., MacGowan, 
A., McLauchlin, J. and Courvalin, P. 1992. Genetic basis of tetracycline resistance in clinical isolates of Listeria monocytogenes. Antimicrob. Agents Chemother. 36: 463-466.

13. Rice, L.B. 1998. Tn916 family conjugative transposons and dissemination of antimicrobial resistance determinants. Antimicrob. Agents Chemother. 42: 1871-1877.

14. Shimoji, Y., Yokomizo, Y., Sekizaki, T., Mori, Y. and Kubo, M. 1994. Presence of a capsule in Erysipelothrix rhusiopathiae and its relationship to virulence for mice. Infect. Immun. 62: 2806-2810.

15. Su, Y.A., He, P. and Clewell, D.B. 1992. Characterization of the $\operatorname{tet}(\mathrm{M})$ determinant of $\operatorname{Tn} 916$ : evidence for regulation by transcription attenuation. Antimicrob. Agents Chemother. 36: 769-778

16. Takahashi, T., Sawada, T., Ohmae, K., Terakado, N., Muramatsu, M., Seto, K., Maruyama, T. and Kanzaki, M. 1984. Antibiotic resistance of Erysipelothrix rhusiopathiae isolated from pigs with chronic swine erysipelas. Antimicrob. Agents Chemother. 25: 385-386.

17. Vicente, M.F., Baquero, F. and Perez-Diaz, J.C. 1988. Conjugative acquisition and expression of antibiotic resistance determinants in Listeria spp. J. Antimicrob. Chemother. 21: 309318.
18. Warsa, U.C., Nonoyama, M., Ida, T., Okamoto, R., Okubo, T., Shimauchi, C., Kuga, A. and Inoue, M. 1996. Detection of tet $(\mathrm{K})$ and tet $(\mathrm{M})$ in Staphylococcus aureus of Asian countries by the polymerase chain reaction. J. Antibiotics (Tokyo) 49: 1127-1132.

19. Wood, R.L. 1992. Erysipelas. pp. 475-486. In: Diseases of Swine, 7th ed. (Lemen, A. D., Straw, B. E., Mengeling, W. L., D'Allaire, S. and Taylor, E. D. J. eds.), Iowa State University Press, Ames.

20. Yamamoto, K., Kijima, M., Yoshimura, H. and Takahashi, T. 2001. Antimicrobial susceptibilities of Erysipelothrix rhusiopathiae isolated from pigs with swine erysipelas in Japan, 1988-1998. J. Vet. Med. B Infect. Dis. Vet. Public Health 48: $115-126$.

21. Yamamoto, K., Sasaki, Y., Ogikubo, Y., Noguchi, N., Sasatsu, M. and Takahashi, T. 2001. Identification of the tetracycline resistance gene, tet(M), in Erysipelothrix rhusiopathiae. J. Vet. Med. B Infect. Dis. Vet. Public Health 48: 293-301.

22. Zilhao, R., Papadopoulou, B. and Courvalin, P. 1988. Occurrence of the Campylobacter resistance gene tet $\mathrm{O}$ in Enterococcus and Streptococcus spp. Antimicrob. Agents Chemother. 32: 1793-1796. 